\title{
RESIDUES AND CHARACTERISTIC CLASSES OF FOLIATIONS
}

\author{
BY JAMES L. HEITSCH
}

Communicated by E. H. Brown, December 2, 1976

In this note we announce results and construct examples which show that a large number of characteristic classes for real foliations vary linearly independently. This generalizes the result of Thurston on the variation of the Godbillon-Vey invariant [T]. The method used is a special case of the general theory of residues of singular foliations due to Baum and Bott $[\mathrm{BB}]$.

Definition. Let $\tau$ be a codimension $q$ foliation on a manifold $M$. A vector field $X$ on $M$ is a $\Gamma$ vector field for $\tau$ if $[X, Y]$ is tangent to $\tau$ whenever $Y$ is tangent to $\tau$. The singular set of $X$ is the set of points where $X$ is tangent to $\tau$.

Let $\tau$ be an oriented codimension $q$ foliation on an oriented manifold $M$. Let $X$ be a $\Gamma$ vector field for $\tau$ and assume the singular set of $X$ consists of a single compact leaf $N$ of $\tau$. On $M-N, \tau$ and $X$ span a foliation $\hat{\tau}$ of codimension $q-1$. Let $\alpha^{*}: H^{*}\left(W O_{q-1}\right) \rightarrow H^{*}(M-N ; R)$ be the natural map associated to $\hat{\tau}$. Each element $\hat{\phi}$ of $H^{2 q-1}\left(W O_{q-1}\right)$ determines in a natural way an element $\phi$ of $H^{2 q}\left(B U_{q} ; R\right)$. Choose an embedded normal sphere bundle $S$ of $N$ in $M$ and let $i: S \rightarrow M-N$ be the inclusion. Denote by $\sigma: H^{2 q-1}(S ; R) \rightarrow H^{q}(N ; R)$ integration over the fiber of the sphere bundle $S$. On $M, \tau$ and $X$ span a singular foliation with singular set $N$. Applying the theory of $[\mathrm{BB}], \phi \in H^{2 q}\left(B U_{q} ; R\right), \tau$ and $X$ determine a cohomology class $\operatorname{Res}_{\phi}(\tau, X, N) \in H^{q}(N ; R)$. We have

THEOREM 1. For $M, N, \tau$, and $X$ as above and $\hat{\phi} \in H^{2 q-1}\left(W O_{q-1}\right)$,

$$
\alpha\left(i * \alpha^{*}(\hat{\phi})\right)=\operatorname{Res}_{\phi}(\tau, X, N) .
$$

Let $\phi \in H^{2 q}\left(B U_{q-1} ; R\right)$. Then $\phi$ and $\hat{\tau}$ determine an element $S_{\phi}(\hat{\tau}) \in$ $H^{2 q-1}(S ; R / Z)$, the Simons' character of $\hat{\tau},[\mathrm{ChS}]$. The element $\phi$ determines in a natural way an element $\phi$ in $H^{2 q}\left(B U_{q} ; R\right)$. We have

THEOREM 2. $S_{\phi}(\hat{\tau})[S]=\operatorname{Res}_{\phi}(\tau, X, N)[N] \bmod Z$, where $[S]$ and $[N]$ are the homology classes determined by $S$ and $N$.

We give some examples which show that these residues are nontrivial and in fact vary linearly independently.

EXAMPLE 1. Denote by $G$ the product of $k$ copies of the special linear group $\mathrm{SL}_{2} R$. Let $K$ be a maximal compact subgroup of $G$ and $\Gamma$ a uniform dis-

AMS (MOS) subject classifications (1970). Primary 57D20, 57D30; Secondary 58D05. 
crete subgroup of $G$ so that $\Gamma \backslash G / K$ is a compact manifold. Let $M$ be the flat $R^{2 k}$ bundle $M=(G / K) \times{ }_{\Gamma} R^{2 k}$ with the natural flat foliation $\tau$. Choose $k$ nonzero numbers $\mu_{1}, \ldots, \mu_{k}$ and let $X$ be the vector field on $R^{2 k}$

$$
X_{\mu}=\sum_{i=1}^{k} \mu_{i}\left(x_{2 i-1} \partial / \partial x_{2 i-1}+x_{2 i} \partial / \partial x_{2 i}\right) .
$$

The natural action of $G$ on $R^{2 k}$ preserves $X_{\mu}$ and so $X_{\mu}$ induces a $\Gamma$ vector field $X_{\mu}$ on $M$ with singular set the zero section $N=\Gamma \mid G / K$. For $\phi \in H^{4 k}\left(B U_{2 k} ; R\right)$ we compute

$$
\operatorname{Res}_{\phi}\left(\tau, X_{\mu}, N\right)=\frac{\pi^{k} \phi\left(\mu_{1}, \mu_{1}, \dot{\mu}_{2}, \mu_{2}, \ldots, \mu_{k}, \mu_{k}\right) \text { vol }}{\left(\mu_{1} \mu_{2} \cdots \mu_{k}\right)^{2}} .
$$

Here vol is a fixed volume form on $N$ and $\phi\left(\mu_{1}, \ldots, \mu_{k}\right)$ is $\phi$, thought of as an invariant polynomial on the lie algebra of the unitary group $U_{2 k}$, applied to the diagonal matrix $\operatorname{diag}\left(\mu_{1}, \mu_{1}, \mu_{2}, \mu_{2}, \ldots, \mu_{k}, \mu_{k}\right)$.

EXAMPLE 2. Let $G$ and $K$ be as in Example 1. We let $G \times R$ act on $R^{2 k+1}=R^{2 k} \times R$ by the natural action of $G$ on $R^{2 k}$ and by the action of $R$ on $R^{2 k+1}$ defined as follows. Let $\omega$ be a smooth, even, nonnegative function on $R$ such that

(i) $0<\omega(x) \leqslant 1$ for all $x \neq 0$.

(ii) For all $x,|x|>1 / 2, \omega(x)=1$.

(iii) $\omega$ and all its derivatives are zero at $x=0$.

On the lie algebra level $R$ acts on $R^{2 k+1}$ by $\partial / \partial t \rightarrow\left|x_{2 k+1}\right| \omega\left(x_{2 k+1}\right) \partial / \partial x_{2 k+1}$. Choose a uniform discrete subgroup $\Gamma$ of $G \times R$ so that $\Gamma \backslash(G \times R) / K$ is a compact manifold. Set $M=(G \times R) / K \times_{\Gamma} R^{2 k+1}$ and let $\tau$ be the natural flat foliation on $M$. Choose nonzero real numbers $\mu_{1}, \ldots, \mu_{k+1}$ and let $X_{\mu}$ be the vector field on $R^{2 k+1}$.

$$
\begin{aligned}
X_{\mu}=\left(\sum_{i=1}^{k} \mu_{i}\left(x_{2 i-1} \partial / \partial x_{2 i-1}+x_{2 i} \partial / \partial x_{2 i}\right)\right) \\
+\mu_{2 k+1} x_{2 k+1} \omega\left(x_{2 k+1}\right) \partial / \partial x_{2 k+1}
\end{aligned}
$$

The action of $G \times R$ on $R^{2 k+1}$ preserves $X_{\mu}$ and so $X_{\mu}$ induces a $\Gamma$ vector field $X_{\mu}$ on $M$ with singular set the zero section $N=\Gamma(G \times R) / K$. For $\phi \in$ $H^{4 k+2}\left(B U_{2 k+1} ; R\right)$ we compute

$$
\operatorname{Res}_{\phi}(\tau, X, N)=\frac{2 \pi^{k} \phi\left(\mu_{1}, \mu_{1}, \mu_{2}, \mu_{2}, \ldots, \mu_{k}, \mu_{k}, 0\right) \text { vol }}{\left(\mu_{1} \mu_{2} \cdots \mu_{k}\right)^{2} \mu_{k+1}} .
$$

As before vol is a fixed volume form on $N$ and $\phi\left(\mu_{1}, \ldots, \mu_{k}, 0\right)$ is $\phi$ applied to the diagonal matrix $\operatorname{diag}\left(\mu_{1}, \mu_{1}, \mu_{2}, \mu_{2}, \ldots, \mu_{k}, \mu_{k}, 0\right)$.

Let $R\left[\sigma_{1}, \ldots, \sigma_{n}\right]$ be the algebra of symmetric polynomials on the variables $\mu_{1}, \ldots, \mu_{n}$ and denote by $R_{m}^{n}$ the subalgebra generated by the elements $\sigma_{i}\left(\mu_{1}, \mu_{1}, \mu_{2}, \mu_{2}, \ldots, \mu_{m}, \mu_{m}, 0, \ldots, 0\right), i=1, \ldots, n-1$. Let $R_{m 0}^{n}$ be the ideal 
in $R_{m}^{n}$ generated by the elements $\sigma_{i}\left(\mu_{1}, \mu_{1}, \mu_{2}, \mu_{2}, \ldots, \mu_{m}, \mu_{m}, 0, \ldots, 0\right)$ where $i=1,3,5, \ldots, 2 t+1$ and $2 t+1=n-2$ or $n-1$. Now set $a(m, n)=$ the dimension of the space of elements of degree $n$ in $R_{m 0}^{n}$, and $b(m, n)=$ the dimension of the space of elements of degree $n$ in $R_{m}^{n}$. Finally set $a(2 k+1)=a(k, 2 k+1)$, $a(2 k)=a(k, 2 k)-1, b(2 k+1)=b(k, 2 k+1)$ and $b(2 k)=b(k, 2 k)-1$. The characteristic classes mentioned in Theorems 1 and 2 come from universal characteristic classes in the $R$ and $R / Z$ cohomology of $B \Gamma_{q}$, the classifying space for codimension $q$ foliations. Combining the examples with Theorems 1 and 2 we see that $B \Gamma_{q}$ has many cohomology classes which vary linearly independently. In particular we may view these classes as maps from the homology of $B \Gamma_{q}$ to $R$ or $R / Z$ and so obtain

THEOREM 3. $H_{2 q+1}\left(B \Gamma_{q} ; Z\right)$ admits epimorphisms onto $R^{a(q+1)}$ and $(R / Z)^{b(2 q+1)}$.

In Example 1, the foliation $\hat{\tau}_{\mu}$, spanned by $\tau$ and $X_{\mu}$ on $M-N$ is transverse to the sphere bundle $M^{0}=G / K \times_{\Gamma} S^{2 k-1}$ provided all the $\mu_{i}$ are close to 1 . We lift this foliation to the bundle over $M^{0}, P=G / K \times_{\Gamma} S O_{2 k}$ (actually the bundle $(\Gamma \backslash G){ }_{K} S O_{2 k}$ ) obtaining a foliation with trivial normal bundle. The projection map $\pi: P \rightarrow M^{0}$ is injective in cohomology in dimension $4 k-1$. Thus we have

THEOREM 4. Let $F \mathrm{~T}_{q}$ be the classifying space for codimension $q$ real foliations with trivial normal bundle. Then $H_{4 k-1}\left(F \Gamma_{2 k-1} ; Z\right)$ admits an epimorphism onto $R^{b(2 k)}$.

\section{REFERENCES}

[BB] P. Baum and R. Bott, Singularities of holomorphic foliations, J. Differential Geometry 7 (1972), 279-342. MR 51 \#14092.

[ChS] J. Cheeger and J. Simons, Differential characters and geometric invariants, Lecture, Amer. Math. Soc. Summer Institute, Stanford, 1973 (unpublished).

[T] W. Thurston, Various lectures.

DEPARTMENT OF MATHEMATICS, UNIVERSITY OF ILLINOIS AT CHICAGO CIRCLE, CHICAGO, ILLINOIS 60680 